\title{
Searching for pulsed emission from XTE J0929-314 at high radio frequencies
}

\author{
M. N. Iacolina ${ }^{1}$, M. Burgay ${ }^{2}$, L. Burderi ${ }^{1}$, A. Possenti ${ }^{2}$, and T. Di Salvo ${ }^{3}$ \\ 1 Università di Cagliari, Dipartimento di Fisica, SP Monserrato-Sestu km 0.7, 09042 Monserrato (CA), Italy \\ e-mail: iacolina@ca.astro.it \\ 2 INAF-Osservatorio Astronomico di Cagliari, Loc. Poggio dei Pini, Strada 54, 09012 Capoterra (CA), Italy \\ 3 Università di Palermo, Dipartimento di Scienze Fisiche ed Astronomiche, via Archirafi 36, 90123 Palermo, Italy
}

Received 25 July 2008 / Accepted 15 January 2009

\section{ABSTRACT}

\begin{abstract}
Aims. The aim of this work is to search for radio signals in the quiescent phase of accreting millisecond X-ray pulsars, in this way giving an ultimate proof of the recycling model, thereby unambiguously establishing that accreting millisecond X-ray pulsars are the progenitors of radio millisecond pulsars.

Methods. To overcome the possible free-free absorption caused by matter surrounding accreting millisecond X-ray pulsars in their quiescence phase, we performed the observations at high frequencies. Making use of particularly precise orbital and spin parameters obtained from X-ray observations, we carried out a deep search for radio-pulsed emission from the accreting millisecond X-ray pulsar XTE J0929-314 in three steps, correcting for the effect of the dispersion due to the interstellar medium, eliminating the orbital motions effects, and finally folding the time series.

Results. No radio pulsation is present in the analyzed data down to a limit of $68 \mu \mathrm{Jy}$ at $6.4 \mathrm{GHz}$ and $26 \mu \mathrm{Jy}$ at $8.5 \mathrm{GHz}$.

Conclusions. We discuss several mechanisms that could prevent the detection, concluding that beaming factor and intrinsic low luminosity are the most likely explanations.
\end{abstract}

Key words. pulsars: general - methods: data analysis - methods: observational - X-rays: binaries - stars: neutron

\section{Introduction}

The neutron star low-mass X-ray binaries (NS-LMXB) are systems containing a neutron star (NS) believed to have a weak magnetic field $\left(B<10^{10} \mathrm{G}\right)$ and accreting matter from a low-mass $\left(M \leq 1 M_{\odot}\right)$ companion. A special subgroup of NS-LMXBs is that of the neutron star soft X-ray transients (NS-SXT; see e.g. White et al. 1995). These systems are usually found in a quiescent state with luminosities in the range $10^{31}-10^{34} \mathrm{erg} \mathrm{s}^{-1}$. On occasion they exhibit outbursts with peak luminosities in the $0.5-10 \mathrm{keV}$ range between $10^{36}$ and $10^{38} \mathrm{erg} \mathrm{s}^{-1}$, during which their behaviour closely resembles that of the persistent NS-LMXBs (Campana et al. 1998). According to the recycling scenario (Alpar et al. 1982; Bhattacharya \& van den Heuvel 1991), the NS-LMXBs are the progenitors of the millisecond radio pulsars (MSPs), reaccelerated by mass and angular momentum transfer from the companion star.

Although widely accepted, there is no direct evidence to confirm this scenario yet. However, in 1998, the idea that NSs in NS-LMXBs are spinning at millisecond periods was spectacularly demonstrated by the discovery of coherent X-ray pulsation at $2.5 \mathrm{~ms}$ in SAX J1808.4-3658, an NS-SXT with an orbital period $P_{\text {orb }} \sim 2$ h (Wijnands \& van der Klis 1998; Chakrabarty \& Morgan 1998). For almost four years, SAX J1808.4-3658 has been considered a rare object in which some peculiarity of the system (e.g. its inclination) allowed detection of the NS spin; however, the situation is now dramatically changed as nine other NS-SXTs in outburst have been discovered in which coherent
$\mathrm{X}$-ray pulsations in the millisecond range have been found (e.g. Wijnands et al. 2006; Krimm et al. 2007; Casella et al. 2008; Altamirano et al. 2008). We are, therefore, facing a new class of astronomical objects (dubbed accreting millisecond X-ray pulsars: AMXPs) that may constitute the bridge between accretionpowered rapidly-rotating and rotation-powered NSs; in particular, the detection of radio pulsations from these sources during quiescence would be the ultimate proof of the validity of the recycling model.

During the mass transfer, the plasma from the companion settles into an accretion disk, whose inner rim is located at a distance $r_{\mathrm{m}}$ from the NS centre, with $r_{\mathrm{m}}$ the so-called magnetospheric radius, which is equal to a fraction $\phi \leq 1$ of the Alfvèn radius, at which the ram pressure of the infalling matter from the accretion disk balances the pressure of the NS magnetic field:

$r_{\mathrm{m}}=1.0 \times 10^{6} \phi \mu_{26}^{4 / 7} \dot{m}_{\mathrm{E}}^{-2 / 7} m_{1}^{-1 / 7} R_{6}^{-2 / 7} \quad \mathrm{~cm}$.

Here $\mu_{26}$ is the magnetic moment in units of $10^{26} \mathrm{G} \mathrm{cm}^{3}, R_{6}$ is the NS radius in units of $10^{6} \mathrm{~cm}, \dot{m}_{\mathrm{E}}$ the Eddington accretion rate in $M_{\odot} \mathrm{yr}^{-1}$ (for $R_{6}=1, \dot{m}_{\mathrm{E}}=1.5 \times 10^{-8} M_{\odot} \mathrm{yr}^{-1}$ and scales with the radius of the compact object), and $m_{1}$ the NS mass in solar masses.

For the radio emission mechanism to switch on in a rotating magnetized NS, the space surrounding the NS must be free of matter up to the light cylinder radius (at which the speed of the 
material co-rotating with the NS would be equal to the speed of light):

$r_{\mathrm{lc}}=5 \times 10^{6} P_{-3} \quad \mathrm{~cm}$

where $P_{-3}$ is the pulse period in milliseconds.

During an outburst, the occurrence, in some cases, of type I bursts and the observation of coherent X-ray pulsation indicate that at the origin of the observed luminosity is the accretion mechanism onto the NS surface. As a consequence coherent radio emission cannot occur in this phase. In the quiescent state, the $0.5-10 \mathrm{keV}$ luminosity is detected at levels ranging between $10^{31.5}$ and $10^{34} \mathrm{erg} \mathrm{s}^{-1}$. Accretion of matter at a lower rate was originally proposed to account for this lower luminosity emission. However detailed studies of the X-ray spectrum in quiescence (Rutledge et al. 2001) and of the thermal relaxation of the NS crust during this phase (Colpi et al. 2001) suggest that the cooling of the periodically warmed up NS surface (Brown et al. 1998) is a viable explanation for the bulk of the luminosity in quiescence. If this is the correct interpretation, there should be no mass accreted onto the neutron star surface during the X-ray quiescent phase, hence $\dot{m} \sim 0$, and thus, plausibly,

$r_{\mathrm{m}} \geq r_{\mathrm{lc}}$.

The timescale for the expansion of the magnetospheric radius beyond the light cylinder radius, in response to a sudden drop in the mass transfer rate, is much shorter (Burderi et al. 2001) than the typical duration (approximately years) of a phase of quiescence in an NS-SXT, allowing the radio pulsar to switch on in principle.

\subsection{Absorption effect of the matter surrounding the system}

In 2001 Burderi et al. proposed a model able to explain the failure of previous and subsequent searches (Burgay et al. 2003, and references therein) for pulsed radio emission from quiescent NSSXTs. As mentioned above, a temporary significant reduction of the mass-transfer rate may cause the magnetospheric radius to overcome $r_{\mathrm{lc}}$, thus allowing a radio pulsar to switch on. In some cases, even if the secular mass-transfer rate is restored, the accretion of matter onto the NS can be inhibited because the radiation pressure from the rotating dipole may be capable of ejecting most of the matter overflowing from the companion out of the system. This phase has been defined as radio-ejection. One of the strongest predictions of this model is the presence, during the radio-ejection phase (hence during X-ray quiescence), of a strong wind of matter emanating from the companion star swept away by the radiation pressure of the pulsar (see Di Salvo et al. 2008a, for a discussion of a possible secular evolution of these systems). This matter, as well as that residual from a previous outburst, can play a role in absorbing the radio signal. Following Burderi et al. (2001) and Burgay et al. (2003), we can estimate the optical depth, $\tau_{\mathrm{ff}}$, at various radio frequencies due to matter engulfing an NS-SXT in its quiescent phase:

$\tau_{\mathrm{ff}}=1.6 \times 10^{3} \times \frac{\gamma^{2} \dot{m}_{-10}^{2}(X+0.5 Y)^{2} F\left(m_{1}, m_{2}\right) \mathrm{Ga}}{m_{1}^{5 / 3} T_{4}^{3 / 2} P_{h}^{4 / 3} v_{9}^{2}\left(1+m_{1} / m_{2}\right)^{5 / 3}}$

where $\dot{m}_{-10}$ is the mass transfer rate in outburst in units of $10^{-10} M_{\odot} \mathrm{y}^{-1}, X$ and $Y$ are the mass fraction of hydrogen and helium respectively, $\gamma$ is the fraction of ionized hydrogen, $T_{4}$ the temperature of the outflowing matter in units of $10^{4} \mathrm{~K}, v_{9}$ the frequency of the radio emission in units of $10^{9} \mathrm{~Hz}, P_{\mathrm{h}}$ is the orbital period in hours, $m_{1}$ and $m_{2}$ are the masses of the NS and its companion in solar masses, $F\left(m_{1}, m_{2}\right)=1-0.462 m_{2} /\left(m_{1}+m_{2}\right)$, $\mathrm{Ga}=\mathrm{Ga}\left(T_{4}, v_{9}, Z\right)=1.00+0.48\left(\log T_{4}-\log Z\right)-0.25 \log v_{9}$ takes into account the dependencies of the Gaunt factor from the temperature, the atomic number $Z$, and the frequency.

One example of a system in the radio-ejection phase is PSR J1740-5340, an eclipsing MSP, with a spin period of $3.65 \mathrm{~ms}$ located in the globular cluster NGC 6397 (D'Amico et al. 2001). For this source $\tau_{\mathrm{ff}}=2.8$ at $v_{9}=1.4$ (with $X=0.7, Y=0.3$, $\gamma=1, T_{4}=1, \mathrm{Ga}=0.94$ for $Z=1.1$ ). This shows that the effect of free-free absorption at $1.4 \mathrm{GHz}$ is important, but not severe, for this system. The radio signal from this PSR is indeed visible at this frequency although it is eclipsed and distorted at many orbital phases (D’Amico et al. 2001). X-ray millisecond pulsars are very similar to PSR J1740-5340, the main difference being the orbital period, which is $\sim 32 \mathrm{~h}$ in the case of PSR J1740-5340 and typically ranges from $40 \mathrm{~min}$ to $\sim 4 \mathrm{~h}$ in the case of the X-ray millisecond pulsars. The difference in the orbital period results in a very different optical depth for the free-free absorption caused by the matter present around these systems. But the dependence of $\tau_{\mathrm{ff}}$ on the square inverse of the radio frequency implies that, at higher frequencies, the effect of the surrounding matter will be less important. For instance, applying Eq. (4) to one of the tighter orbits AMXPs, XTE J0929-314, at $1.4 \mathrm{GHz}$, at $6.4 \mathrm{GHz}$ and at $8.5 \mathrm{GHz}$, with parameters $\dot{m}_{-10} \sim 2.9, m_{1} \sim 1.4 M_{\odot}$, $m_{2} \sim 0.02 M_{\odot}, X=0.7, Y=0.3, \gamma \sim 1, T_{4} \sim 1, P_{\mathrm{h}}=0.73$ (Galloway et al. 2002 ) leads to $\tau_{\mathrm{ff}}(1.4 \mathrm{GHz}) \approx 5, \tau_{\mathrm{ff}}(6.5 \mathrm{GHz}) \approx$ $0.2, \tau_{\mathrm{ff}}(8.5 \mathrm{GHz}) \approx 0.1$. Adopting these two observing frequencies, the problem of the absorption of the radiation is totally overcome. Prompted by these considerations, we have undertaken a programme to search for millisecond pulsations in NSSXT XTE J0929-314 at 6.5 and 8.5 GHz.

The observations and the method of data analysis are presented in Sect. 2, and the results are reported in Sect. 3 and discussed in Sect. 4.

\section{Observations and data analysis}

Radio observations of the millisecond pulsar XTE J0929-314 were made with the Parkes $64 \mathrm{~m}$ radio telescope. Three data series were taken on 2003 December 19-21 with a bandwidth of $576 \mathrm{MHz}$. The first was at a central radio frequency of $6410.5 \mathrm{MHz}$, the other two at a central radio frequency of 8453.5 MHz.

The collected signal for each polarization was split into 192 $3 \mathrm{MHz}$ channels using an analogue filterbank, in order to minimize the pulse broadening caused by dispersion in the interstellar medium (ISM). The outputs from each channel were one bit digitized every $100 \mu \mathrm{s}$. The resulting time series were stored on digital linear tapes (DLT) for off-line analysis. The observations lasted $7.5 \mathrm{~h}$ each, corresponding to $2^{28}$ samples. We then rebinned the data to obtain $2^{27}$ samples in order to reduce the computational time; therefore, the effective time resolution of the analysed data is $200 \mu \mathrm{s}$.

The data analysis methodology was chosen on the basis of the precise knowledge of the orbital and spin parameters for XTE J0929-314, obtained from the X observation. The original ephemerides were presented by Galloway et al. (2002), while we used those reported in Table 1, which have been recently refined by Di Salvo et al. (in prep.; see also Di Salvo et al. 2008b).

Off-line analysis was made by means of a software suite that, in the first stage, aimed to reduce the dispersion effects on the signal due to the interstellar medium. The data series were 
Table 1. Orbital and spin parameters for XTE J0929-314 (Di Salvo et al., in prep.; see also Di Salvo et al. 2008b).

\begin{tabular}{|c|c|}
\hline Parameter & $\overline{\text { Value }}$ \\
\hline RAJ & $09^{\mathrm{h}} 29^{\mathrm{m}} 20^{\mathrm{s}} .19$ \\
\hline DecJ & $-31^{\circ} 23^{\prime} 3^{\prime \prime} 2$ \\
\hline Orbital period, $P_{\text {orb }}(\mathrm{s})$ & 2614.748(3) \\
\hline Projected semi-major axis, $a \sin i$ (lt-ms) & $5.988(10)$ \\
\hline Eccentricity, $e$ & $<0.007$ \\
\hline Spin period $^{a}, P_{\mathrm{S}}(\mathrm{s})$ & $0.0054023317856(4)$ \\
\hline Mean spin period derivative, $\dot{P}_{\mathrm{S}}\left(\mathrm{s} \mathrm{s}^{-1}\right)$ & $1.63(12) \times 10^{-18}$ \\
\hline Ascending node passage, $T_{0}(\mathrm{MJD})$ & $52405.48676(1)$ \\
\hline
\end{tabular}

${ }^{a}$ The value reported is referred at epoch 52 396.5 MJD.

The errors on the last quoted digit(s) are intended to be at $1 \sigma$ and are reported in parentheses.

dedispersed according to 72 trial dispersion-measure (DM) values ranging from 5.51 to $396.74 \mathrm{pc} \mathrm{cm}^{-3}$ for the data series at $6410.5 \mathrm{MHz}$ and 33 trial $\mathrm{DM}$ values ranging from 12.64 to $416.99 \mathrm{pc} \mathrm{cm}^{-3}$ for the data series at $8453.5 \mathrm{MHz}$. The maximum DM was chosen to be $\sim 4$ times the nominal DM value obtained using either the Taylor \& Cordes (1993) or the Cordes \& Lazio (2001) models for the distribution of free electrons in the ISM, in order to account for the errors in the estimated distance of the source, for the uncertainties in the ISM model, and for the presence of local matter surrounding the system, hence increasing the local DM (estimated to be at most $\sim 100 \mathrm{pc} / \mathrm{cm}^{3}$ for this source; Burgay et al. 2003).

The successive step is to deorbit and barycentre the time series, i.e. to eliminate the effects of the orbital motion of the NS in the binary system and the earth in the solar system. This was obtained by resampling the time series in order to mimick time series collected from a telescope located in the barycentre of the solar system and looking at the source as if it were located at the barycentre of the pulsar orbit.

In doing this exercise it is important to estimate the effect on the putative pulsar signal due to the uncertainties in the adopted ephemeris for the system. Therefore we simulated dedispersed time series containing a periodic signal with the spin and orbital characteristics of XTE J0929-314, as derived by Di Salvo et al. (Table 1). We then deorbited and barycentred these time series using the upper and the lower limits of the parameters in Table 1, adopting $1 \sigma$ errors.

It turned out that even a variation in all the parameters (but the orbital period, see later) at $1 \sigma$ level does not affect the detectability of the pulsations, since that produced a maximum broadening of the pulse much smaller than 0.1 in pulsar phase. The only exception is for the orbital period: $1 \sigma$ variation in $P_{\text {orb }}$, propagated over the $\sim 19500$ orbits occurred between X-ray and radio observations, produces a broadening of 0.4 in pulsar phase for our observation. Therefore, to obtain a maximum broadening of the pulse of 0.1 in phase, we corrected the time series using 8 trial values of $P_{\text {orb }}$ ( 4 above and 4 below the nominal value), covering the $1 \sigma$ uncertainty range.

The third step in the procedure was to fold the (already deorbited and barycentred) time series according to the rotational parameters reported in Table 1 . The trial values of the spin period used for the folding, were chosen taking into account that the nominal value of $P_{\mathrm{S}}$ dates from the X-ray observations of May 2002, while our observations occurred 19 months later. Therefore we explored a range of spin period $\Delta P_{\text {tot }}=$ $P_{\max }-P_{\text {min }}$, with $P_{\text {max }}=P_{\mathrm{S}}+\delta P_{\mathrm{S}}+\Delta T\left(\dot{P}_{\mathrm{S}}+\delta \dot{P}_{\mathrm{S}}\right)$ and $P_{\text {min }}=$ $P_{\mathrm{S}}-\delta P_{\mathrm{S}}$, where $P_{\mathrm{S}}$ and $\dot{P}_{S}$ are the values reported in Table 1 , $\delta P_{\mathrm{S}}$ and $\delta \dot{P}_{\mathrm{S}}$ their $1 \sigma$ errors, and $\Delta T$ is the time between the observations in X-ray and radio bands. As a first guess we used the outburst value of $\dot{P}_{\mathrm{S}}$ for this calculation, resulting a safe upper limit for the dipolar spin-down ${ }^{1}$, as shown below. For a safer investigation, we also checked on spin period values lower than our previous estimate $P_{\min }$ and for the double of the nominal value, $2 \times P_{\mathrm{S}}$.

Since the spin period derivative measured in outburst is usually different from that in the quiescent phase, we checked the plausibility of the adopted trial period interval through an estimate of the surface magnetic field $B_{\mathrm{S}}$. This estimate can be obtained evaluating the magnetic torque acting on the NS through the formula (Rappaport et al. 2004; Di Salvo et al., in prep.):

$\tau(t)=\dot{M}(t) \sqrt{G M R_{\mathrm{CO}}}-\frac{\mu^{2}}{9 R_{\mathrm{CO}}^{3}}$

where $\dot{M}$ is the mass accretion rate, $M$ the NS mass, $\mu$ the magnetic dipole moment, $R_{\mathrm{CO}}$ the corotation radius (at which the disc matter in Keplerian motion rotates with the same angular speed of the NS) in cgs units. Since this AMXP is observed to spin down, the contribution to the spin up from the accretion (first additive term in 5) is neglected. The torque can also be written:

$\tau(t)=2 \pi I \dot{v}_{\mathrm{S}}(t)$

where $I$ is the NS moment of inertia and $\dot{v}_{\mathrm{S}}$ the spin frequency derivative. Since $\dot{v}_{\mathrm{S}}$ and $R_{\mathrm{CO}}$ are known and, assuming $I=$ $10^{45} \mathrm{~g} \mathrm{~cm}^{2}$, we can obtain the value of the magnetic moment and, in turn, an estimate of $B_{\mathrm{S}}$. Then, through the relation

$B=3.2 \times 10^{19} \sqrt{P \dot{P}_{\text {dip }}} \quad$ Gauss,

we can obtain an estimate of the dipolar spin-down $\dot{P}_{\text {dip }}$ during observation (in quiescence), assuming the spin down is governed by magnetodipole braking. It leads to $B_{\mathrm{S}}=6.6 \times 10^{8}$ Gauss and $\dot{P}_{\text {dip }}<\dot{P}_{\mathrm{S}}$ and then we can conclude that the adopted $\Delta P_{\text {tot }}$ is safely large. Therefore, since the pulse broadening for an error equal to $1 / 20$ of the $\Delta P$ interval is less than 0.1 in phase over our observations, we selected 40 trial values of the period, 20 to the right and 20 to the left of the nominal value to cover the $\Delta P_{\text {tot }}$ interval calculated above.

A further check of the validity of the derived $B_{\mathrm{S}}$ (hence of the adopted $\Delta P_{\text {tot }}$ ) can be derived from optical observations (Monelli et al. 2005; D'Avanzo et al. 2008). In fact, the optical counterpart of the XTE J0929-314 companion observed in December 2003 (very close to our radio observations) with the VLT has a luminosity one order of magnitude higher than expected. This luminosity excess can be interpreted as the luminosity $L_{\mathrm{PSR}}$ isotropically irradiated by the rotating magneto-dipole, intercepted, and reprocessed by the companion star, as observed e.g. by Burderi et al. (2003) and Campana et al. (2004) for SAX J1808.4-3658, and by D'Avanzo et al. (2007) for IGR J00291+5934.

The excess luminosity can be written as the fraction $f=$ $f_{\mathrm{C}}+f_{\mathrm{D}}$ of dipolar radiation intercepted by the companion star and the disk (see Burderi et al. 2003):

$L_{\mathrm{exc}}=f \cdot L_{\mathrm{PSR}}=f \cdot \frac{2 \mu^{2} \omega^{4}}{3 c^{3}}=f \cdot 3.85 \times 10^{35} P_{-3}^{-4} \mu_{26}^{2} \mathrm{erg} \mathrm{s}^{-1}(8)$

where $\omega$ is the rotational frequency of the NS and $f_{\mathrm{C}}$ can be written as $f_{\mathrm{C}}=2 \pi a^{2}(1-\cos \theta) /\left(4 \pi a^{2}\right)$, where $a$ is the the orbital

\footnotetext{
1 The expected variation of the $P_{\mathrm{S}}$ during the radio observation is negligible and hence does not affect a correct folding.
} 

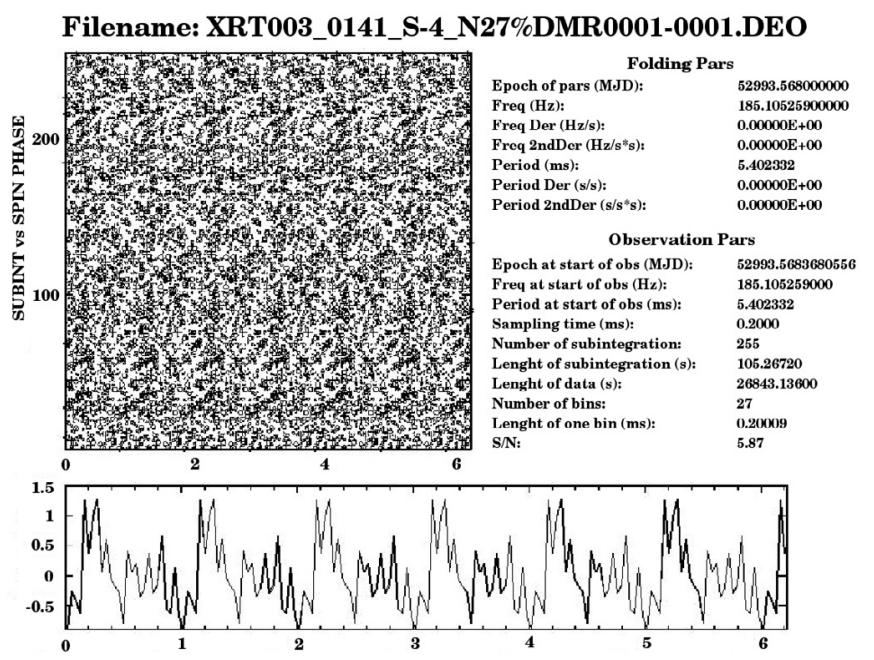

Fig. 1. Example of a plot resulting from the procedure described in the text. The bottom diagram shows six integrated pulse profiles, while the grayscale on the left represents the signal in the 255 subintegrations in which the observation has been subdivided. On the right, the parameters used for the folding are shown, along with the parameters of the observation.

separation and $\theta$ the angle subtended by the companion star as seen from the central source. If the companion star fills its Roche lobe, this can be written as $\sin \theta=R_{\mathrm{L} 2} / a$, where $R_{\mathrm{L} 2}$ is the Roche lobe radius of the secondary and $R_{\mathrm{L} 2} / a=0.49 q^{2 / 3} /\left[0.6 q^{2 / 3}+\right.$ $\left.\ln \left(1+q^{1 / 3}\right)\right]$ (Eggleton 1983). Assuming a mass ratio of $q=$ $m_{2} / m_{2}=0.02 / 1.4$ and $\sin i=1$, we obtain $f_{\mathrm{C}}=0.003$; while $f_{\mathrm{D}}=0.012$ is evaluated adopting a standard Shakura-Sunyaev disk model (Shakura \& Sunyaev 1973) with $\dot{m}_{-10}=2.9$, and is given by the projected area of the disk as seen by the central source, $2 \pi R \times 2 H(R)$ (where $R$ is the disk outer radius and $H(R)$ the disk semi-thickness at $R$ ), divided by the total area, $4 \pi R^{2}$.

Adopting the value of $L_{\text {exc }} \simeq 3.33 \times 10^{32} \mathrm{erg} \mathrm{s}^{-1}$ reported by Monelli et al. (2005) and $f \simeq 1.15 \times 10^{-2}$, we can thus derive $B_{\mathrm{S}} \simeq 7 \times 10^{8}$ Gauss, in good agreement with the value estimated above.

\section{Results}

Spanning the searched ranges of $P_{\text {orb }}, P_{\mathrm{S}}$ and DM we have obtained 50922 plots, reporting the result from the folding of the deorbited and barycentred time series. An example of these plots (obtained from the observation at $6.5 \mathrm{GHz}$ ) is shown in Fig. 1. The bottom diagram shows the integrated pulse profile (6 phases), while the grayscale on the left represents the signal in the 255 subintegrations in which the observation has been subdivided. A good candidate should display a roughly linear trend in the grayscale and a high signal-to-noise ratio pulse profile. On the right, the parameters used for the folding of this candidate are shown, along with the parameters of the observation.

A useful diagnostic tool that can be adopted to further evaluate the plausibility of a suspect is shown in Fig. 2, where a grayscale of the strength of the signal (with darker points at higher $\mathrm{S} / \mathrm{N}$ ), plotted as a function of dispersion measure DM and spin frequency $v_{\mathrm{S}}$, should define a clear peak around the correct parameters of the putative pulsar. The same kind of plot can be created at a constant DM with $v_{\mathrm{S}}$ and $P_{\text {orb }}$ on the axes or at a constant $v_{\mathrm{S}}$, tracing the $S / N$ trend at varying DMs and $P_{\text {orb }}$.
Table 2. Parameters for the pulsar candidate with the highest $S / N$ shown in Fig. 1.

\begin{tabular}{lc}
\hline \hline Signal to noise ratio, $S / N$ & 5.87 \\
Dispersion Measure, DM $\left(\mathrm{pc} \mathrm{cm}^{-3}\right)$ & 5.51 \\
Orbital period, $P_{\text {orb }}(\mathrm{s})$ & 2614.74575 \\
Projected semi-major axis, $a$ sin $i(\mathrm{lt}-\mathrm{ms})$ & $6.290(9)$ \\
Spin frequency, $v_{\mathrm{S}}(\mathrm{Hz})$ & 185.105259 \\
Ascending node passage, $T_{0}(\mathrm{MJD})$ & $52405.48676(1)$ \\
\hline
\end{tabular}

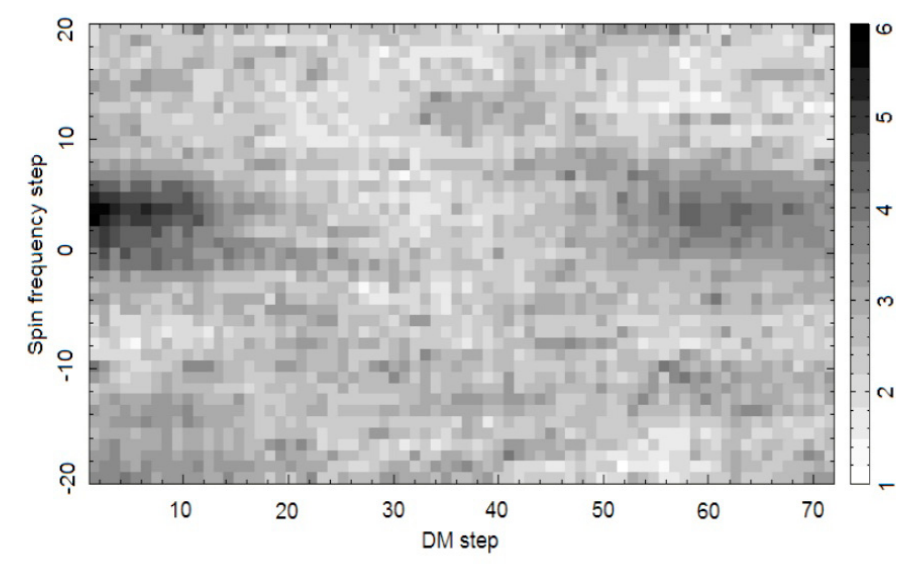

Fig. 2. Signal-to-noise ratio in function of the spin frequency (20 step above and 20 below the nominal value, corresponding to 0 ) and the dispersion measure (72 steps corresponding to values interval from 5.51 to $\left.396.74 \mathrm{~cm}^{-3} \mathrm{pc}\right)$ at $P_{\text {orb }}=2614.74575 \mathrm{~s}$. The highest point corresponds to the best $S / N, \approx 6$, reported in Fig. 1 .

The plots in Figs. 1 and 2 refer to the suspect with the highest $S / N$ found in our search. Its folding parameters are listed in Table 2. The peak in the profile has a $3.4 \sigma$ significance that, over the 26568 trial foldings performed on the $6.5 \mathrm{GHz}$ dataset (72 DMs $\times 9 P_{\text {orb }} \times 41 P_{\mathrm{S}} \mathrm{s}$ ), has a probability of not being randomly generated by noise of $\sim 10^{-6}$. We also note that, in Fig. 2 the decreasing $S / N$ trend is not particularly defined, although a maximum is present. Also, that the DM that maximises the $S / N$ is close to zero (w.r.t an expected value of $\gtrsim 100 \mathrm{pc} / \mathrm{cm}^{3}$ ) weakens the credibility of the signal. Finally, this signal suspect is not confirmed in the observations at higher frequency $(8.5 \mathrm{GHz})$, elaborated with the same parameters. We can conclude that no radio pulsation with the expected periodicity has been found in the source XTE J0929-314 during its quiescent phase.

\subsection{Flux density upper limits}

A rough estimate of the flux density for a pulsar of period $P$ is (e.g. Manchester et al. 1996)

$S=\epsilon n_{\sigma} \frac{T_{\text {sys }}+T_{\text {sky }}}{G \sqrt{N_{\mathrm{p}} \Delta t \Delta v_{\mathrm{MHz}}}} \sqrt{\frac{W_{\mathrm{e}}}{P-W_{\mathrm{e}}}} \quad \mathrm{mJy}$,

where $n_{\sigma}=6.0$ is the threshold (in unit of sigma) for having a statistically significant signal, given the number of performed foldings; $T_{\text {sys }}=50 \mathrm{~K}$ the system noise temperature for the observations at $\sim 6 \mathrm{GHz}$ and $T_{\text {sys }}=25 \mathrm{~K}$ for those at $\sim 8 \mathrm{GHz}$ (see Parkes website: http://www.parkes.atnf.csiro.au/ observing/documentation); $T_{\text {sky }}$ is the sky temperature in Kelvin, calculated from that at $v=408 \mathrm{MHz}$ and considering a scaling with the frequency as $v^{-2.7} ; G$ is the gain of the radio 


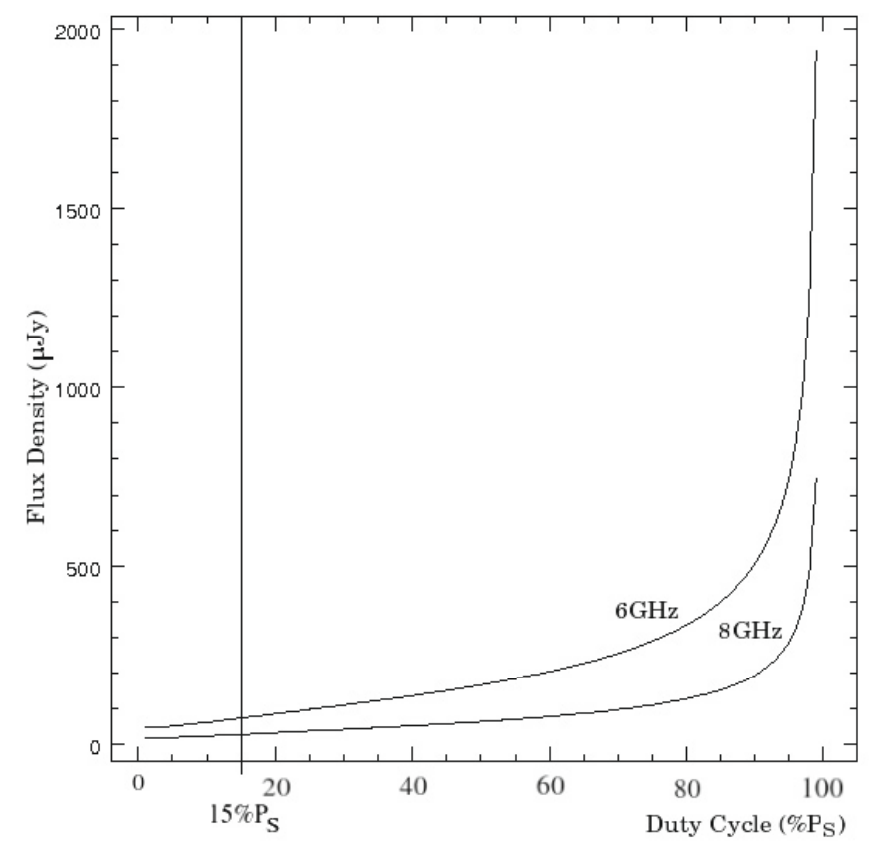

Fig. 3. Flux density upper limits in $\mu \mathrm{Jy}$ for XTE J0929-314 obtained with Eq. (9) for increasing values of the duty cycle.

telescope (in $\mathrm{K} \mathrm{Jy}^{-1}$ ), with 0.46 for the observations at $\sim 6 \mathrm{GHz}$ and 0.59 for those at $\sim 8 \mathrm{GHz}$, (see Parkes website); $\Delta t$ is the integration time in seconds, $N_{\mathrm{p}}$ the number of polarizations (here 2), and $\Delta v_{\mathrm{MHz}}=576 \mathrm{MHz}$ is the bandwidth in $\mathrm{MHz}$. The term $\epsilon \sim 1.4$ is a factor accounting for the sensitivity reduction due to digitisation and other losses. The term $W_{\mathrm{e}}$ is the effective width of the pulse:

$W_{\mathrm{e}}=\sqrt{W^{2}+(\beta \delta t)^{2}+\delta t_{\mathrm{DM}}^{2}+\delta t_{\mathrm{scatt}}^{2}}$.

Its value depends on the sampling time ( $\delta t=200 \mu \mathrm{s}$ in this case), on the technical characteristics of the receiver $(\beta=2)$, on the broadening of the pulse introduced by both the dispersion of the signal in each channel $\left(\delta t_{\mathrm{DM}} \sim 4 \div 9 \times 10^{-8} \times \mathrm{DM} \mathrm{s}\right.$, depending on the frequency), on the scattering induced by inhomogeneities in the ISM $\left(\delta t_{\text {scatt }} \sim 10^{-9} \mathrm{~s}\right)$, and on the intrinsic pulse width $W$. The dependence of the minimum flux density achievable from the duty cycle, $W / P$, is shown in Fig. 3. A duty cycle of $15 \%$ of the spin period is assumed and, with this value, the flux density upper limits for XTE J0929-314 are: $S_{\mathrm{MAX}}^{6.5}=68 \mu \mathrm{Jy}$ at $6410.5 \mathrm{MHz}$ and $S_{\text {MAX }}^{8.5}=26 \mu \mathrm{Jy}$ at $8453.5 \mathrm{MHz}$.

\section{Discussion}

In the hypothesis that a radio pulsar was on during our observations, we examine several possible explanations for the lack of the detection of radio pulsation from our source.

\subsection{The luminosity}

In Fig. 4 the pseudoluminosity $L=S \cdot d^{2}$ in $\mathrm{mJy} \mathrm{kpc}^{2}$ (where $S$ is the measured flux density and d the distance of the source) distribution at $1.4 \mathrm{GHz}$ for a sample of 42 galactic field MSPs is shown ${ }^{2}$. For XTE J0929-314 the upper limits on the

2 These values are derived from the ATNF catalogue - http://www . atnf.csiro.au/research/pulsar/psrcat/; Manchester et al. (2005).

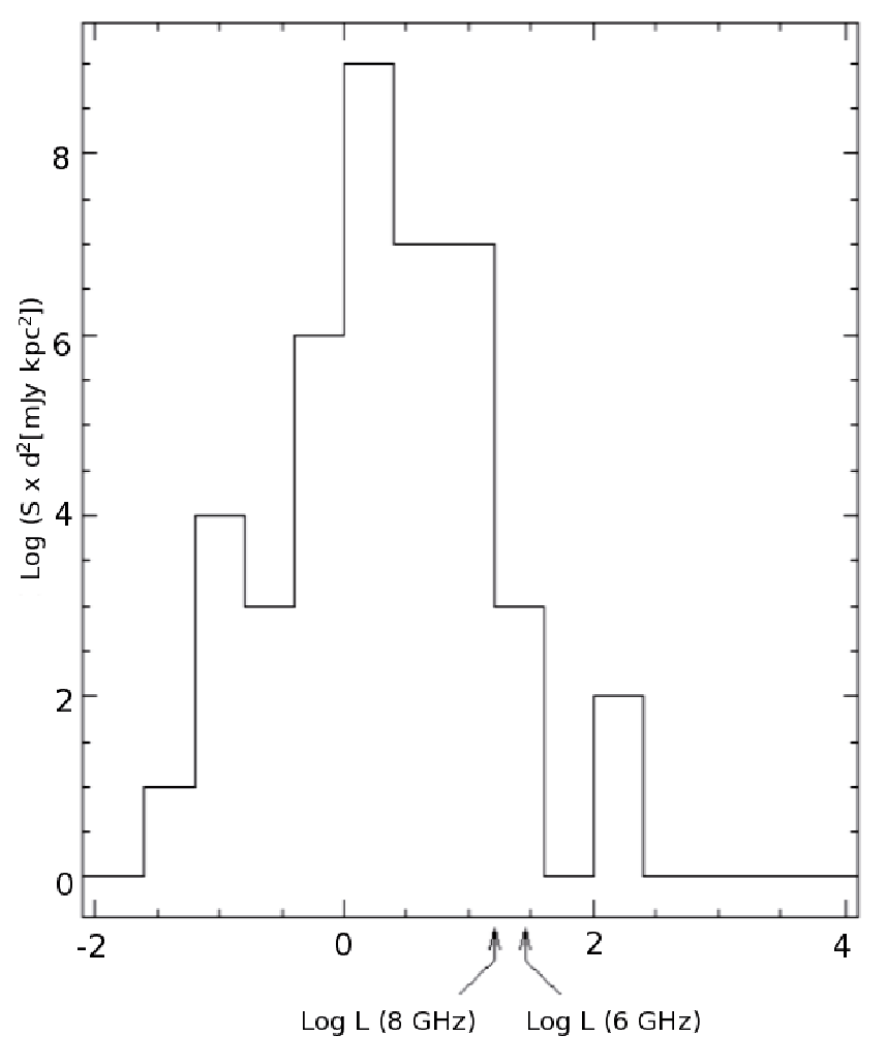

Fig. 4. Pseudoluminosity distribution of a sample of 42 field MSPs. The arrows indicate the upper limits of the pseudoluminosity scaled at 1.4 GHz for XTE J0929-314, derived on the basis of the minimum flux detectable and assuming a distance of $6 \mathrm{kpc}$ (Galloway et al. 2002).

pseudoluminosity has been calculated assuming a distance of $6 \mathrm{kpc}$ (Galloway et al. 2002) and our flux density upper limits at 6.4 and $8.5 \mathrm{GHz}$ scaled to $1.4 \mathrm{GHz}$ assuming a spectral index for MSPs of 1.7 (Kramer et al. 1998). As a result, about $90 \%$ of the known MSPs are below the pseudoluminosity upper limits of XTE J0929-314.

This suggests that XTE J0929-314 in quiescence might not be a very bright MSP and that a deeper search should be carried out to sample lower values of the flux density. However, given the distance of XTE J0929-314 (significantly higher than the typical distance of the sample of known MSPs), only nextgeneration instruments, like the Square Kilometer Array, will be able to discover pulsed radio emission from this source at the high frequencies adopted in this work, if its luminosity is at the faintest end of the known distribution.

\subsection{The beaming factor}

The emission from a pulsar is strongly anisotropic. This means that it does not irradiate in the same way in all directions, but only on one usually narrow portion of sky, which can be quantified through the so-called beaming factor. Therefore, the lack of a detection of a radio signal could be due to unfavourable geometry of the radio emission with respect to the observer.

The average value, $f(\alpha)$, of the fraction of the sky swept from two conal radio beams of half width $\alpha$ is (Emmering \& Chevalier 1989):

$f(\alpha)=\int_{0}^{\pi / 2} f(\alpha, \eta) \sin \eta \mathrm{d} \eta=(1-\cos \alpha)+\left(\frac{\pi}{2}-\alpha\right) \sin \alpha$ 
where $\eta$ is the angle (supposed randomly distributed) between the magnetic axis (aligned with the radio beams) and the rotational axis, and $f(\alpha, \eta)=\cos [\max (0, \eta-\alpha)]-\cos [\min (0, \eta+\alpha)]$.

Considering a $10 \% \div 30 \%$ interval of width of the pulse, it follows that $9^{\circ} \leq \alpha \leq 27^{\circ}$ and, therefore, using Eq. (11), $0.23 \leq$ $f(\alpha) \leq 0.61$. In particular, assuming $15 \%$ typical value of width of the impulse, the probability that the radio emission cone does not intersect our line of sight is $66 \%$, with $f(\alpha) \approx 0.34$.

\section{Conclusions}

The aim of this work was to search for radio pulsations from the AMXP XTE J0929-314. The detection of radio signals in the quiescent phase of this kind of transient systems would be ultimate proof of the recycling model, unambiguously establishing that the AMXPs are the progenitors of radio MSP. No radio pulsation has been detected in the analysed data down to a limit of $68 \mu \mathrm{Jy}$ at $6.4 \mathrm{GHz}$ and $26 \mu \mathrm{Jy}$ at $8.5 \mathrm{GHz}$. Assuming that a radio pulsar was on during the observation, the free-free absorption cannot be responsible for the lack of a detection, given the relatively high radio frequencies. The beaming factor is a viable explanation for that, since the probability of any unfavourable geometry of radio emission with respect to the observer is $\sim 60 \div 70 \%$. However, the most likely reason for a negative result is that the source has a luminosity lower than our limits. In fact, more than $90 \%$ of the known MSPs show a luminosity lower than the upper limits that we have derived for XTE J0929-314.

To get round to these problems, one can make observations at an intermediate frequency between 6.5 and $1.4 \mathrm{GHz}$. For a maximum mass transfer rate in quiescence equal to the outburst value, we can estimate that the lower limit in frequency for obtaining $\tau_{\mathrm{ff}} \sim 1$ is $\sim 3 \mathrm{GHz}$. Calculating $S_{\mathrm{MAX}}$ for an observation with all the same parameters (adopted in the present work) but the frequency (now set at $3 \mathrm{GHz}$ ), we could sample more than a half of the known MSPs luminosity distribution.

Acknowledgements. A.P. and M.B. acknowledge the financial support to this research provided by the Ministero dell' Istruzione, dell'Università e della Ricerca (MIUR) under the national programme PRIN052005024090_002.

\section{References}

Alpar, M. A., Cheng, A. F., Ruderman, M. A., \& Shaham, J. 1982, Nature, 300, 728

Altamirano, D., Casella, P., Patruno, A., Wijnands, R., \& van der Klis, M. 2008, ApJ, 674, L45

Bhattacharya, D., \& van den Heuvel, E. P. J. 1991, Phys. Rep., 203,

Brown, E. F., Bildsten, L., \& Rutledge, R. E. 1998, ApJ, 504, L95

Burderi, L., Possenti, A., D’Antona, F., et al. 2001, ApJ, 560, L71

Burderi, L., Di Salvo, T., D’Antona, F., Robba, N. R., \& Testa, V. 2003, A\&A, 404, L43

Burgay, M., Burderi, L., Possenti, A., et al. 2003, ApJ, 589, 902

Campana, S., Colpi, M., Mereghetti, S., Stella, L., \& Tavani, M. 1998, A\&ARv, 8,279

Casella, P., Altamirano, D., Patruno, A., Wijnands, R., \& van der Klis, M. 2008, ApJ, 674, L41

Chakrabarty, D., \& H., M. E. 1998, Nature, 394, 346

Colpi, M., Geppert, U., Page, D., \& Possenti, A. 2001, ApJ, 548, L175

Cordes, J. M., \& Lazio, T. J. W. 2001, ApJ, 549, 997

D'Amico, N., Possenti, A., Manchester, R. N., et al. 2001, ApJ, 561, L89

D’Avanzo, P., Campana, S., Casares, J., et al. 2008, AIP Conf. Ser., 1068, 217

Di Salvo, T., Burderi, L., Riggio, A., Papitto, A., \& Menna, M. T. 2008a, MNRAS, 389, 1851

Di Salvo, T., Burderi, L., Riggio, A., Papitto, A., \& Menna, M. T. 2008b, AIP Conf. Ser., 1054, 173

Eggleton, P. P. 1983, ApJ, 268, 368

Emmering, R. T., \& Chevalier, R. A. 1989, ApJ, 345, 931

Galloway, D. K., Chakrabarty, D., Morgan, E. H., \& Remillard, R. A. 2002, ApJ, 576, L137

Kramer, M., Xilouris, K. M., Lorimer, D. R., et al. 1998, ApJ, 501, 270

Krimm, H. A., Markwardt, C. B., Deloye, C. J., et al. 2007, ApJ, 668, L147

Manchester, R. N., Lyne, A. G., D’Amico, N., et al. 1996, MNRAS, 279, 1235

Manchester, R. N., Hobbs, G. B., Teoh, A., \& Hobbs, M. 2005, VizieR Online Data Catalog, 7245, 0

Monelli, M., Fiorentino, G., Burderi, L., et al. 2005, in Interacting Binaries: Accretion, Evolution, and Outcomes, ed. L. Burderi, L. A. Antonelli, F. D’Antona, et al., AIP Conf. Ser., 797, 565

Rappaport, S. A., Fregeau, J. M., \& Spruit, H. 2004, ApJ, 606, 436

Rutledge, R. E., Bildsten, L., Brown, E. F., Pavlov, G. G., \& Zavlin, V. E. 2001, ApJ, 559, 1054

Shakura, N. I., \& Syunyaev, R. A. 1973, A\&A, 24, 337

Taylor, J. H., \& Cordes, J. M. 1993, ApJ, 411, 674

White, N. E., Nagase, F., \& Parmar, A. N. 1995, in X-ray binaries, ed. W. H. G. Lewin, J. van Paradijs, \& E. P. J. van den Heuvel (Cambridge University Press), 1

Wijnands, R. 2006, in BAAS, 38, 1183

Wijnands, R., \& van der Klis, M. 1998, Nature, 394, 344 